\title{
Beyond biomarkers: an opportunity to address the 'pharmacodynamic gap' in pediatric drug development
}

\begin{abstract}
"It is now the time to apply 'out-of-the-box' thinking to develop, evaluate and validate new approaches to assess drug effect (PD) in infants and children that are scientifically valid and appropriate for use in the segment of the population that they are intended to benefit."
\end{abstract}

\section{KEYWORDS: biomarkers $\approx$ pediatrics $\approx$ pharmacodynamic}

As recently denoted by Wagner, a biomarker is defined as "A characteristic that is measured and evaluated as an indicator of normal biologic processes, pathogenic processes or pharmacological responses to a therapeutic intervention" [1]. While it is widely recognized that children are not simply small adults with regard to disease expression and its response to therapy with drugs or biologics, the aforementioned definition of 'biomarker' is equally applicable to adults and children.

\section{"One might effectively argue that the promise of personalized medicine for pediatric and adult patients will rely greatly on the development and use of appropriate biomarkers: tests/tools that will be characterized by accuracy and precision in their ability to inextricably link disease detection with an ability to predict treatment success/failure..."}

Biomarkers are increasingly being integrated into the practice of clinical pediatrics. When one considers some of the more common biomarkers currently in use, they represent the spectrum from classic (e.g., hemoglobin A1C and anti-dsDNA) through to molecular (e.g., soluble CD13 and genotype for drug metabolizing enzymes), with capabilities ranging from diagnostic capacity through to evaluating disease progression, treatment response and, in some instances, the ability to individualize drug therapy on an a priori basis (e.g., genotyping for thiopurine methyltransferase, CYP2C9 and VKORC1). One might effectively argue that the promise of personalized medicine for pediatric and adult patients will rely greatly on the development and use of appropriate biomarkers: tests/tools that will be characterized by accuracy and precision in their ability to inextricably link disease detection with an ability to predict treatment success/failure, and enable optimization through greater adaptive control (i.e., fine tuning) of the exposure-response relationship for drugs and, potentially, biologics. However, it is clear that their utility lies beyond a patient-specific realm.

As summarized by Wagner et al. [2], both the 'Road Map' initiative of the NIH and the 'Critical Path' initiative from the US FDA have called for an expanded application of biomarker technology to the arenas of drug development and regulatory decision-making. These initiatives have spawned an accelerated evolution in biomarker development by providing a discernable and desirable target (i.e., commercialization) for their creation, development, use, validation and, ultimately, regulation [3]. For example, some biomarkers can be qualified as surrogate end points that link biological processes with clinical end points [4] and, in some instances, are intended to substitute for a clinical end point [101] so as to facilitate the drug approval process. To some degree, this appears to have been the case with some 90 new drug applications receiving accelerated approval by the FDA from 1992 to 2008 that were based on surrogate end points [101]. In addition, from January 1998 to June 2008, the FDA approved 69 applications for new molecular entities on the basis of surrogate end points, which were considered as valid substitutes for the demonstration of clinical benefit from a given drug. Unfortunately for children, much of this work has focused on treatments for diseases that have preponderance in adults (e.g., HIV/AIDS, cancer and Type 2 diabetes) [4]. This is not to say that the priorities that must drive drug development in adults occur at the expense of children but, rather, to highlight the potential for surrogate end points to fill a critical void in pediatric drug development. Specifically, biomarkers must

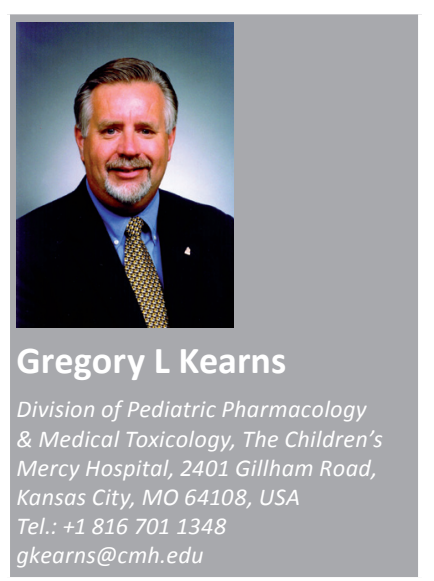

future medictio $^{\text {pisg }}$ fs 
be exploited for their potential utility as a tool for characterizing the impact of development (i.e., ontogeny) on drug action and effect.

Recent commentaries on surrogate end points and their qualification clearly indicate that a biomarker's value resides in either an association with clinical end points [2] and/or outcome resulting from a therapeutic intervention [5]. Given that most surrogate end points are grounded by biologic plausibility and that many used to guide therapeutic decision-making have an association with either a drug's mechanism(s) of action and/or an established (usually in adults) concentration-effect relationship, it is plausible that the utility of biomarkers can be extended so as to create a bridge between the ascertainment of drug effect and the prediction of efficacy in pediatric patients. This assertion is supported by consideration of the current 'Pediatric Decision Tree,' which is part of the Agency's guidance document on exposure-response relationships [102] and is currently used by the FDA to guide determinations on the types of studies that pharmaceutical companies must conduct in accordance with the provisions of existing legislation regulating pediatric drug development (e.g., Best Pharmaceuticals for Children Act and the Pediatric Research Equity Act). As illustrated in Figure 1, sole reliance upon bridging pharmacokinetic $(\mathrm{PK})$ and safety trials for pediatric drug labeling can only be permitted in accordance with current FDA guidance [102]

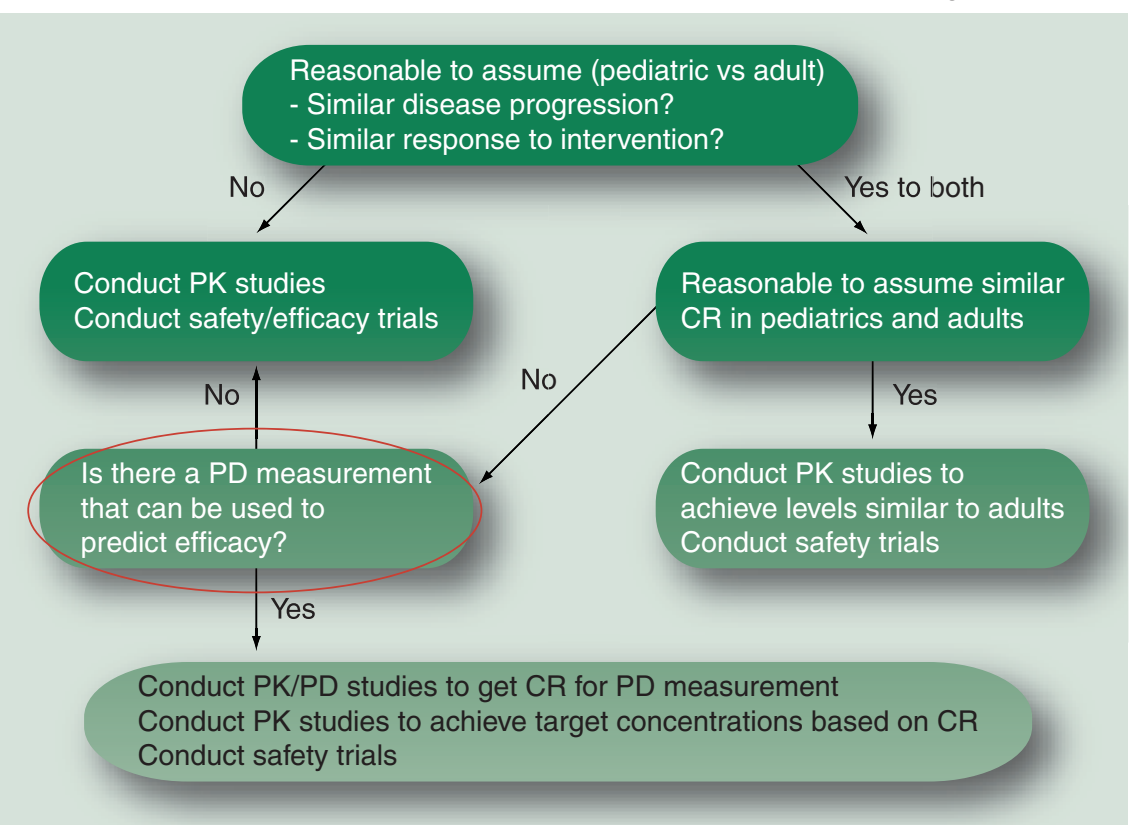

Figure 1. US FDA pediatric tree. Importance of PD measurement to the decisionmaking promise is denoted by the red circle.

CR: Concentration response; PD: Pharmacodynamic; PK: Pharmacokinetic.

Adapted from [102]. when it is reasonable to assume that both pediatric and adult patients demonstrate similar disease progression and response to therapeutic intervention (including similarity in concentrationresponse relationship). Clear precedent for such an approach is seen with sotalol, an antiarrhythmic agent approved for ventricular tachycardia in adults, using recurrence of atrial fibrillation and flutter as end points. As recently reviewed by Gobburu [5], this drug was subsequently approved for use in pediatric patients on the basis of $\mathrm{PK}$ and exposure-response data relating to electrocardiographic changes (QTc interval) and heart rate. Similarly, piperacillin/tazobactam (an antimicrobial drug product) was approved for pediatric use primarily based upon PK data, which enabled the determination of age-appropriate doses required to achieve systemic exposures, similar to those demonstrated in adults, to be associated with drug efficacy.

Despite the relative dearth of information from human clinical trials conducted over the last decade, which objectively demonstrates an association between ontogeny and pharmacodynamic (PD) $[6,7]$, it is generally assumed that both drug disposition and action exhibit a significant developmental dependence. The importance of this PD 'information gap' was heralded in a recent commentary by Holford, who proclaimed that "testing for PK without testing for PD in children is unethical" [8]. While the tone of this declaration could be interpreted as somewhat nihilistic with respect to the documented importance of pediatric PK data for improving drug treatment, at the heart of the comment is profound truth. Namely, to truly understand the relationship between human development and both drug disposition and action, and to translate this knowledge into 'directions' sufficient for a practitioner to use for the purpose of age-specific optimization of drug treatment, it is essential that the process of pediatric drug development equally embraces PK and PD. To realize this necessary goal will require new approaches that are capable of transcending the two greatest obstacles, to date, in quantifying drug concentration-effect relationships in infants and children:

- The lack of validated (in children), dynamic end points that are capable of accurately measuring the drug-receptor interaction;

- Availability of end points that are both child and parent 'friendly' (e.g., noninvasive, do not produce discomfort and can be performed in the context of clinical patient care). 
The Holy Grail in overcoming these obstacles resides with the creation of $\mathrm{PD}$ surrogate end points that can accurately and reproducibly measure drug effect in infants and children, and, by inference, constitute a measurement that can be used to reliably predict drug efficacy (Figure 1). A survey of several recent publications suggests that work to develop PD surrogate end points suitable for use in pediatric patients is underway.

"Owing to innovations in regulation,
regulatory science and clinical/translational
science, we remain in the midst of a 'perfect
storm' with regard to pediatric drug
development. Many improvements have
been made ... all to the benefit of increased
understanding and translation of important
new information into the context of
pediatric therapeutics."

Building upon work previously performed in adults [9], Neville et al. demonstrated that specific pupillary measurements in children provide a method that can be used to study the PD of analgesic drugs [10]. Jones et al. have recently reported results from a study that used laser Doppler flowimetry to measure microvascular blood flow as a means to describe the time-dependent PD of histamine; a study which has immediate application for the study of antihistamine clinical pharmacology in infants and children by using an integrated $\mathrm{PK} / \mathrm{PD} /$ pharmacogenomic strategy [11]. In addition, Jones and colleagues have demonstrated the utility of the ${ }^{13} \mathrm{C}$-acetate breath test as a dynamic measurement of gastric emptying in pediatric patients [12]; a technique with great potential as a functionally appropriate (as opposed to traditional studies that measure intraesophageal $\mathrm{pH}$ ) PD surrogate end point that can be used to study the impact of ontogeny on the exposure-response relationships of prokinetic agents intended for the treatment of gastroesophageal reflux in children. It is worth noting that all three of these recent examples of pediatric PD surrogate end points completely overcome the aforementioned 'obstacles' for assessing drug action in pediatric patients.

Owing to innovations in regulation, regulatory science and clinical/translational science, we remain in the midst of a 'perfect storm' with regard to pediatric drug development. Many improvements have been made and, as a result, knowledge has been expanded; all to the benefit of increased understanding and translation of important new information into the context of pediatric therapeutics. However, significant challenges remain in the arena of assessing PD in children. Specifically, if we continue to use our current paradigms for determining the requisites of a pediatric drug development plan (FIGURE 1), we risk propagation of practice, which has the potential to limit the rate at which new drugs can be introduced and, ultimately, the benefit to children throughout the world. In this context, two relevant quotations from great thinkers immediately come to mind:

- "We can't solve problems by using the same kind of thinking we used when we created them" (Albert Einstein).

- "There can be no keener revelation of a society's soul than the way in which it treats its children" (Nelson Mandela).

It is now the time to apply 'out-of-the-box' thinking to develop, evaluate and validate new approaches to assess drug effect (PD) in infants and children that are scientifically valid and appropriate for use in the segment of the population that they are intended to benefit. The responsibility for this resides with all who are involved across the continuum of drug development, regulation and use. An avenue that can enable this successful journey resides with the continued development of biomarkers so as to extend their utility consistent with their potential to inform us about the success or failure of drug treatment. The race begins now.

\section{Disclosure}

The opinions expressed therein are those of the author and do not reflect the position of the Children's Mercy Hospital, the University of Missouri - Kansas City or any entity with whom the author has a professional or personal affliation.

Financial \& competing interests disclosure

The author is supported, in part, by grant number 5U10HD031313-17 (Pediatric Pharmacology Research Unit Network) and contract HHSN2752010000031 (Pediatric Trial Network) from the Eunice Kennedy Shriver National Institute of Child Health and Human Development, Bethesda, MD, USA. No financial support was received by the author and/or his employer (the Children's Mercy Hospital) for the preparation of this commentary. The author has no other relevant affiliations or financial involvement with any organization or entity with a financial interest in or financial conflict with the subject matter or materials discussed in the manuscript apart from those disclosed.

No writing assistance was utilized in the production of this manuscript. 


\section{Bibliography}

1 Wagner JA: Biomarkers: principles, policies and practice. Clin. Pharmacol. Ther. 86, 3-7 (2009).

2 Wagner JA, Williams SA, Webster CJ: Biomarkers and surrogate end points for fit-for-purpose development and regulatory evaluation of new drugs. Clin. Pharmacol. Ther. 81, 104-107 (2007).

3 Wilson C, Schulz S, Waldman SA: Biomarker development, commercialization, and regulation: individualization of medicine lost in translation. Clin. Pharmacol. Ther. 81, 153-155 (2007).

4 Lathia CD, Amakye D, Dai W et al.: The value, qualification and regulatory use of surrogate end points in drug development. Clin. Pharmacol. Ther. 86, 32-43 (2009).

5 Gobburu JVS: Biomarkers in clinical drug development. Clin. Pharmacol. Ther. 86, 26-27 (2009).

6 Kearns GL, Abdel-Rahman SM, Alander SW, Blowey DL, Leeder JS, Kauffman RE: Developmental pharmacology - drug disposition, action and therapy in infants and children. N. Engl. J. Med. 349, 1157-1167 (2003).

7 Mulla H: Understanding developmental pharmacodynamics: importance for drug development and clinical practice. Paediatr. Drugs 12, 223-233 (2010).

8 Holford N: Dosing in children. Clin. Pharmacol. Ther. 87, 367-370 (2010).

9 Ibrahim AE, Feldman J, Karim A, Kharasch ED: Simultaneous assessment of drug interactions with low- and highextraction opioids: application to parecoxib effects on the pharmacokinetics and pharmacodynamics of fentanyl and alfentanil. Anesthesiology 98, 853-861 (2003).

10 Neville KA, Anderson R, Griggs M, Peter S, Connelly M: Pupillometry: a tool to measure pharmacodynamic effect of analgesic drugs in pediatrics. Clin. Pharmacol. Ther. 87, S65-S66 (2010).

11 Jones BL, Abdel-Rahman SM, Simon SD, Kearns GL, Neville KA: Assessment of histamine pharmacodynamics by microvascular response of histamine using histamine iontophoresis laser doppler flowimetry. J. Clin. Pharmacol. 49, 600-605 (2009).

12 Jones BL, Pearce RE, Abdel-Rahman SM, Friesen CA, James LP, Kearns GL:

Characterization of delayed gastric emptying in children by the ${ }^{13} \mathrm{C}$-acetate breath test. J. Breath Res. 3, 1-6 (2009).

\section{Websites}

101 New drug approval: FDA needs to enhance its oversight of drugs approved on the basis of surrogate endpoints. United States government accountability office (Report GAO-09-866), September 2009 www.gao.gov/products/GAO-09-866

102 Guidance for industry: Exposure-response relationships - study design, data analysis, and regulatory applications. US department of health and human services, food and drug administration, center for drug evaluation and research and center for biologics evaluation and research, April 2003 www.fda.gov/downloads/Drugs/ GuidanceComplianceRegulatoryInformation/ Guidances/ucm072109.pdf (Accessed 12 October 2010) 